\title{
Management of Paediatric Head Injuries in Sweden: A 5 National Cross-Sectional Survey
}

Fredrik Wickbom ( $\nabla$ fredrik.wickbom@gmail.com )

Lund University https://orcid.org/0000-0002-2274-2887

\section{Linda Persson}

Region Halland

\section{Zandra Olivecrona}

Örebro University Hospital

Johan Undén

Lunds University Faculty of Medicine

Original research

Keywords: mTBI, TBI, children, guidelines, initial management, Sweden, descriptive

Posted Date: September 7th, 2021

DOl: https://doi.org/10.21203/rs.3.rs-868274/v1

License: (c) (1) This work is licensed under a Creative Commons Attribution 4.0 International License. Read Full License 


\section{Management of paediatric head injuries in Sweden: a}

9 Authors

$10 \quad$ Full names

11 Fredrik Wickbom*1,2, Linda Persson*3, Zandra Olivecrona ${ }^{4}$ and Johan Undén ${ }^{1,2}$

12 Institutional addresses

$13{ }^{1}$ Department of Operation and Intensive Care, Hallands Hospital, Halmstad, Sweden

$14 \quad{ }^{2}$ Lund University, Lund, Sweden

$15{ }^{3}$ Department of Orthopaedics, Hallands Hospital, Halmstad, Sweden

$16{ }^{4}$ Department of Neurosurgery, Faculty of Health and Medicine, Department for Medical

17 Sciences, Örebro, Sweden

18 Corresponding author

19 Fredrik Wickbom fredrik.wickbom@regionhalland.se

$20 *$ *Fredrik Wickbom and Linda Persson contributed equally to this work. 


\section{Abstract}

\section{Background}

3

4 Previous studies have shown varying management of children with traumatic brain injury

5 (TBI) in Sweden. Recently, new guidelines have been introduced which may have affected

6 management of these patients.

\section{$7 \quad$ Methods}

8 Cross-sectional structured survey, containing different management domains, in Sweden

9 during 2020, using an on-line survey system aiming to describe initial (first 24 hours)

10 management of TBI in children. Data presented as descriptive analysis and comparisons with

11 Fisher exact test when applicable.

\section{Results}

1356 hospitals of differing size were included in the analysis (response rate 93\%). 76\% used

14 established guidelines. Children with TBI are predominately managed by inexperienced

15 doctors (84\%), primarily from non-paediatric specialities (75\%). Most hospitals (75\%) have

16 the possibility to observe children with TBI and almost all have complete access to CT scans.

17 In larger hospitals, it was more common for nurses to discharge patients without doctor

18 assessment $(\mathrm{p}<0.001)$. Use of established guidelines and written observation routines has

19 increased significantly since $2006(\mathrm{p}<0.001)$.

\section{Conclusions}

21 Management of children with TBI still varies in Sweden, although many aspects have significantly improved over the last 15 years. Most hospitals use established guidelines, 
1 utilise dose-reduction protocols for CT, use written observation routines and provide

2 adequate information to patients/guardians at discharge.

\section{Keywords}

5 mTBI; TBI; children; guidelines; initial management; Sweden; descriptive

\section{Background}

8 Traumatic brain injury (TBI) is recognized as a common cause of death and disability among

9 children worldwide [1]. The incidence varies; a recent review on global epidemiology of TBI

10 in children and adolescents showed that $691 / 100000$ children per year were treated in

11 emergency departments (ED's), 74/100 000 was admitted to hospital and median mortality

12 rate was 9/100 000 children and year [2]. Most patients (70-98\%) are classified as having

13 minimal or mild traumatic brain injury (mTBI) primarily from their initial level of

14 consciousness (Glasgow Coma Scale, GCS) with or without specific risk factors [3-6]. Two

15 thirds of these patients will swiftly recover without suffering from any persisting sequelae

$16[7,8]$. However, a minority will suffer life-threatening intracranial haemorrhages in need of

17 urgent attention and often rapid surgical intervention [9]. The task of effectively identifying

18 these patients is a clinical challenge.

19

20 Computed tomography (CT) is the gold standard used to detect intracranial complications

21 after TBI, such as intracranial haemorrhages. CT is readily available in the western world and

22 relatively inexpensive and simple to use. However, as CT utilizes ionizing radiation, a scan 
1 increases the patient's lifetime risk of cancer; a risk even more pronounced in the paediatric

2 population which [10] warrant careful consideration when subjecting children to CT scans

$3 \quad[11,12]$.

4 In-hospital observation is an alternative management approach to detect complications after

5 mTBI [5]. Evolving data support in-hospital observation as an equally valid strategy in terms

6 of clinical outcome [13]. Evaluation of risks and benefits, regarding aspects such as costs,

7 practicality and radiation issues, need to be performed in decision making for every

8 individual case. In 2006, a national survey regarding management of paediatric mTBI

9 revealed inconsistencies in the care of this patient group [14]. According to this study, only

$1027 \%$ of hospitals in Sweden used any sort of guideline for management of paediatric TBI.

11 Initial assessment in emergency departments was primarily performed by non-specialists

12 (assistant residents and/or residents) in $96 \%$ of cases [14]. This aspect is important, as

13 attributes of the treating doctor has shown to influence the frequency of diagnostic imaging,

14 with more risk -intolerant physicians more likely to order a CT scan, compared to colleges

15 with greater risk tolerance [15]. Decisions concerning diagnostic tests are also influenced by

16 concerns about patient satisfaction and malpractice issues, with substantiation knowledge and

17 decision support frequently requested $[16,17]$.

19 Differences in management routines and lack of established guidelines may negatively affect 20 patient safety. The use of clinical decision rules (CDR's) for management of mTBI has been 21 shown to decrease the number of CT scans without an elevated risk of missing potentially 22 dangerous intracranial haemorrhages $[11,18]$. Several evidence-based guidelines for 23 management of mTBI in children have been derived to provide decision-making support 24 regarding the utilization of head CT scans. Among the most commonly cited are the 25 Paediatric Emergency Care Applied Research Network (PECARN) head CT rule [9], the 
1 Canadian Assessment of Tomography of Childhood Head Injury-rule (CATCH) [19] and

2 Children's Head injury Algorithm for the prediction of Important Clinical Events-rule

3 (CHALICE) [20]. However, none of these CDRs are developed with the Scandinavian health

4 care system in consideration. Also, concerns regarding a potential increase in CT scanning in

5 children with the use of these guidelines have been raised [5]. An existing Scandinavian

6 guideline, published in 2000, was primarily designed for management of adults [21].

7 Therefore, a new guideline (SNC16) was developed and published by the Scandinavian

8 Neurotrauma Committee in 2016 [5] with the Scandinavian health care system in mind. The

9 guideline was produced through a rigorous process of evidence analysis and includes

10 consensus aspects to bridge evidence gaps. This guideline has a somewhat different approach

11 than PECARN, CATCH and CHALICE. It is developed to detect intracranial complications

12 after TBI in need of neurosurgery or medical intervention, and risk stratifies patients in five

13 groups with accompanying recommendations concerning need for CT and/or observation. It

14 also includes recommendations regarding observation time and quality, discharge advice and

15 criteria for later CT scanning. External validation has shown encouraging results [22] and

16 internal, national validation is currently in progress. Recently, another evidence- and

17 consensus-based guideline for mild and moderate head injuries was published by the

18 PREDICT collaborative, specifically developed for usage in Australia and New Zeeland [23],

19 very much in line with the SNC16. How publication of these CDRs has influenced

20 management of children with head injuries in Sweden is unknown.

22 Studies have shown that introducing new guidelines can be difficult [24]. One factor

23 associated with successful implementation is that the guideline is well adapted and applicable

24 in the clinical setting [25]. Prior to introducing the SNC16 nationally in Sweden, knowledge

25 about current practice is of great important in order to optimize the implementation strategy 
1 and achieve a high level of compliance. A previous national survey study [14], which showed

2 considerable variations in management, allows analysis of changes in management over time.

3 Also, although the SNC16 has not been officially implemented, the guideline may be in

4 clinical use due to the lack of accepted alternative options.

5 The aim of this study is therefore to describe current management of these children in

6 Sweden. Secondary aims are to analyse differences in management over time, to assess the

7 implementation of the SNC16 and to analysis possible variations in care.

\section{Methods}

9 This is a descriptive cross-sectional study including all emergency departments at hospitals

10 with the possibility of in-hospital care. Data was collected using a web-based survey designed

11 in collaboration with members of the Scandinavian Neurotrauma Committee (SNC). The

12 survey was designed to answer the most crucial questions regarding the current management

13 of children with TBI in Sweden. The questions were structured into 5 different sections (table

14 1), exemplifying questions from each part. The questionnaire was completed using the

15 program esMaker (Entergate AB). A primary pilot version was sent to 6 hospitals and after

16 minor details adjusted the complete form was finished and sent.

17

18

Table 1. The questionnaire - sections and exemplified main questions

Section 1: Background information

- Name of hospital

- Presence of written guidelines concerning initial management of mTBI in children within 24 hours of trauma 
Section 2: Initial treatment in the emergency department

- What clinic is responsible for paediatric patients with mTBI?

- Are these patients cared for by specialists or non-specialists?

Section 3: Radiology

- What primary radiology modality is used?

- Access to anaesthesiologist and diagnostic radiology

Section 4: In-hospital observation

- What department are patients admitted to in need of in-hospital observation?

- What parameters are being monitored during hospitalization?

Section 5: Discharge and follow-up

- Are patients and guardians provided with discharge information?

- Does your hospital arrange follow up?

2 The survey was answered once per participating hospital. Initial contact was established by

3 phone to ensure that a suitable responder, able to provide valid information and an overall

4 view, was reached. When appropriate recipients had been identified, the questionnaire was

5 distributed by e-mail. Data was collected continuously from June 2020 to March 2021.

6 Reminders were sent by e-mail twice to non-responders until $>90 \%$ response rate was

7 reached.

8 Data is summarized and presented using descriptive statistics. A cross-comparison was

9 performed between four categories depending on the size of the hospital; local, regional,

10 university and children's hospital. Further statistical analysis was performed using Fisher's 
1 exact test to detect differences between groups, when indicated. A two-tailed p-value of

$2<0.05$ was considered significant. Due to known small numbers in the last two groups, the

3 categories were a priori dichotomised to smaller hospitals (local and regional) and larger

4 hospitals (university and children's hospitals). As answers for a certain question were not

5 always $100 \%$ complete, the total number of responses is given with each question.

6 Only the overall management of children with mild head injury was collected in this study.

7 Neither personal data nor individual patient records were obtained. An ethical advisory

8 opinion was granted by the Swedish Ethical Review Authority, Dnr 2020-02693.

\section{Results}

10 Initial contact was established with 76 hospitals in Sweden; 5 hospitals did not manage acute

11 TBI at all. Of the remaining 71 hospitals, responses were returned from 66 hospitals (overall

12 response rate $93 \%) .56$ of these hospitals managed paediatric patients with TBI (10 only

13 managing adult TBI) and form the base of this study. The size and type of the included

14 hospitals $(\mathrm{n}=56)$ varied; 28 local hospitals, 19 regional hospitals, 4 dedicated children's

15 hospitals and 5 university hospitals. Of the 5 non-responding hospitals, 2 were local hospitals,

162 were regional hospitals and one was a university hospital (figure 1). 
$1 \quad$ Figure 1. Flowchart describing participating and non-participating Swedish hospitals

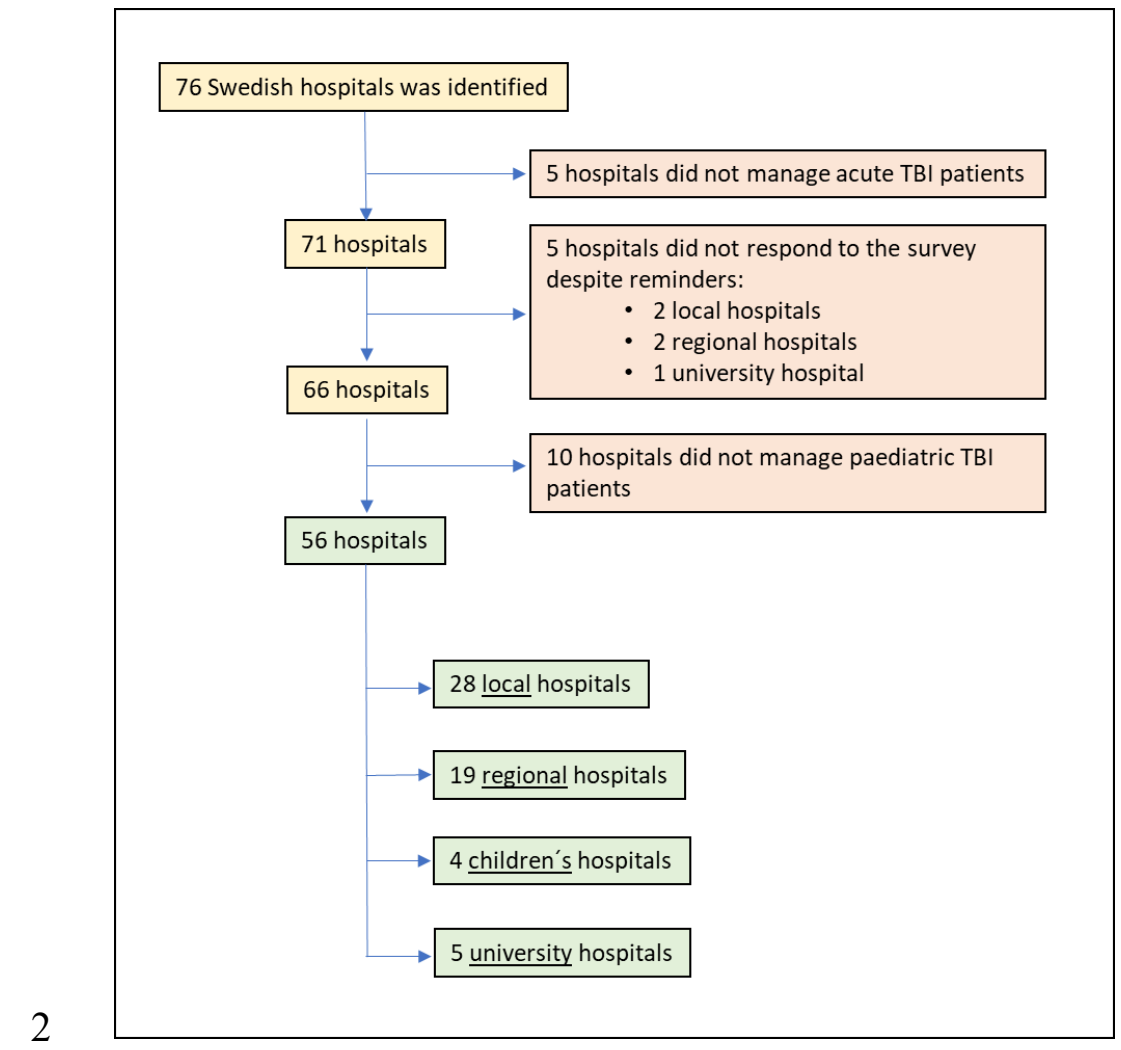

3

4 The age span which was used by participating units to define the patients as paediatric

5 differed. Most common span at the non-paediatric hospitals were 0-18 years of age. In

6 contrast, children's hospitals had a different definition, with the upper limit differing between

$7 \quad 14-16$ years of age.

8 Nine (16\%) hospitals reported limited access to a neurosurgical unit, with a transfer time of $>$

92 hours to the closest neurosurgical service.

10

11 In total, $76 \%(42 / 55)$ of the respondents reported use of an established guideline for the

12 management of paediatric TBI at their hospital (Table 2). When comparing smaller hospitals

13 (local and regional) to larger ones (university and children's), there was no statistically

14 significant difference in presence of guidelines $(\mathrm{p}=0.18)$. The most commonly used guideline

15 was the SNC16 guideline $(n=31,55 \%)$. Following this, the most commonly described was a 
1 local modification of pre-existing validated guidelines (such as PECARN) or local guidelines

2 based on local expert opinion.

4 Table 2. Use of guideline for management of paediatric TBI

\begin{tabular}{|llllll|}
\hline & $\begin{array}{l}\text { Local } \\
\text { Hospital } \\
\mathrm{n}(\%)\end{array}$ & $\begin{array}{l}\text { Regional } \\
\text { Hospital } \\
\mathrm{n}(\%)\end{array}$ & $\begin{array}{l}\text { Children's } \\
\text { Hospital } \\
\mathrm{n}(\%)\end{array}$ & $\begin{array}{l}\text { University } \\
\text { Hospital } \\
\mathrm{n}(\%)\end{array}$ & Total \\
\hline Established guideline & $20(71)$ & $13(68)$ & $4(100)$ & $5(100)$ & $42(76)$ \\
\hline No guideline & $7(25)$ & $4(21)$ & 0 & 0 & $11(20)$ \\
\hline Unknown & 0 & $2(11)$ & 0 & 0 & $2(4)$ \\
\hline Total & $27(96)$ & $19(100)$ & $4(100)$ & $5(100)$ & $55(98)$ \\
\hline
\end{tabular}

5

$6 \quad$ Numbers presented for respective hospital category and all hospital categories in total. Respondent from one

7 (n=1) local hospital did not respond to this question (response rate 55/56, 98\%).

8

9 Children with TBI were predominantly managed by non-specialists (Table 3). There was no

10 statistical difference in the presence of specialists between small (local and regional) and

11 large (university and children's) hospitals $(\mathrm{p}=0.17)$. Apart from dedicated children's hospitals,

12 children with TBI were rarely treated by a doctor with a paediatric speciality (Table 4). Large

13 (university and children's) hospitals had significantly higher presence of paediatric

14 specialities than small (local and regional) hospitals $(\mathrm{p}<0.001)$. 
$1 \quad$ Table 3. Level of experience of responsible clinician.

\begin{tabular}{|lllllll} 
& $\begin{array}{l}\text { Local } \\
\text { Hospital } \\
\mathrm{n}(\%)\end{array}$ & $\begin{array}{l}\text { Regional } \\
\text { Hospital } \\
\mathrm{n}(\%)\end{array}$ & $\begin{array}{l}\text { Children's } \\
\text { Hospital } \\
\mathrm{n}(\%)\end{array}$ & $\begin{array}{l}\text { University } \\
\text { Hospital } \\
\mathrm{n}(\%)\end{array}$ & Total \\
\hline $\begin{array}{l}\text { Non-specialist } \\
\text { "assistant physician, dependent", } \\
\text { "assistant physician, independent", } \\
\text { "intern" and "resident" are merged }\end{array}$ & $22(79)$ & $18(95)$ & $3(75)$ & $4(80)$ & $47(84)$ \\
\hline \begin{tabular}{l} 
Specialist \\
\hline
\end{tabular} & $10(36)$ & $3(16)$ & $3(75)$ & $3(60)$ & $19(34)$ \\
\hline
\end{tabular}

2

3 Most common experience level for clinicians manging children with TBI. Respondent were asked to rate how

often a child with TBI in their ED was managed by a physician with experience level corresponding to “assistant physician, dependent”, “assistant physician, independent”, “intern”, "resident” and "specialist”. For each "category" (experience level) of physician the respondent rated on a 5-grade scale (always; often; sometimes; rarely; never) how frequent this category manage children with head trauma at their emergency department. A dichotomisation of the experience levels to "specialist" and "non-specialist level” (in which categories: "assistant physician, dependent", "assistant physician, independent", "intern" and "resident" were merged) was done for the analysis. To further simplify presentation, grade "always" and "often" was merged (implying the "most common" experience level for clinicians manging children with TBI) and presented for respective hospital size. This means that if grade "sometimes", "rarely" or "never" was chosen for a category (experience level) of physician it won't be presented in the table. Merging of experience levels and response options means that the aggregated total response rate won't be 100\%. Percentages are calculated as number of responses per total hospitals in each category. Example: There was in total 22 responses in the nonspecialist category deriving from local hospitals, implying that in 22 of the 28 local hospitals (79\%) it is common ("often" or "always") that non-specialists are managing children with TBI.

Table 4. Responsible clinic: Paediatric vs non-paediatric specialities

\begin{tabular}{|llllll|} 
& $\begin{array}{l}\text { Local } \\
\text { Hospital } \\
\mathrm{n}(\%)\end{array}$ & $\begin{array}{l}\text { Regional } \\
\text { Hospital } \\
\mathrm{n}(\%)\end{array}$ & $\begin{array}{l}\text { Children's } \\
\text { Hospital } \\
\mathrm{n}(\%)\end{array}$ & $\begin{array}{l}\text { University } \\
\text { Hospital } \\
\mathrm{n}(\%)\end{array}$ & $\begin{array}{c}\text { Total } \\
\mathrm{n}(\%)\end{array}$ \\
\hline Paediatric speciality & 0 & $1(5)$ & $4(100)$ & $2(40)$ & $7(13)$ \\
\hline Non-paediatric speciality & $22(79)$ & $18(95)$ & 0 & $2(40)$ & $42(75)$ \\
\hline Emergency medicine & $10(36)$ & $6(32)$ & 0 & $3(60)$ & $19(34)$ \\
\hline
\end{tabular}


1 Departments responsible for initial management of paediatric head trauma patients displayed for each hospital

2 size and total. Responsible clinic (speciality of the clinic) is categorized as paediatric (paediatric surgery;

3 paediatrics; paediatric neurology; paediatric orthopaedics), non-paediatric (neurology; general surgery;

4 internal medicine; orthopaedics or other speciality) or emergency medicine. For each type of clinic, respondent

5 was asked to rate on a 5-grade scale (always; often; sometimes; rarely; never) how frequent this speciality

6 manages children with head trauma at their emergency department. To further simplify presentation, grade

7 "always" and "often" was merged (implying the "most common" clinic/speciality manging children with TBI)

8 and presented for respective hospital size. This means that if grade "sometimes", "rarely" or "never" was

9 chosen for a clinic/speciality it won't be presented in the table. Merging of clinics/specialities and response

10 options means that the aggregated total response rate won't be 100\%. Percentages are calculated as number of

11 responses per total hospitals in each category. Example: There was in total 10 responses in the emergency

12 medicine category deriving from local hospitals, implying that in 10 of the 28 local hospitals (36\%) it is

13 common ("often" or "always") that emergency medicine physicians are managing children with TBI.

15 As shown in table 4, most of the patients are managed by physicians in non-paediatric specialities (75\%), of which general surgery represents $71 \%(n=40)$. In $34 \%(n=19)$ of the

17 hospitals, emergency medicine physicians often or always manage paediatric patients with

18 TBI. It was uncommon (5\%) that initial assessment always or often was done by a doctor 19 specialising in neurology (Table 5).

Table 5. Responsible clinic: Neurology vs non-neurology specialities

\begin{tabular}{|llllll|} 
& $\begin{array}{l}\text { Local } \\
\text { Hospital } \\
\mathrm{n}(\%)\end{array}$ & $\begin{array}{l}\text { Regional } \\
\text { Hospital } \\
\mathrm{n}(\%)\end{array}$ & $\begin{array}{l}\text { Children's } \\
\text { Hospital } \\
\mathrm{n}(\%)\end{array}$ & $\begin{array}{l}\text { University } \\
\text { Hospital } \\
\mathrm{n}(\%)\end{array}$ & $\begin{array}{c}\text { Total } \\
\mathrm{n}(\%)\end{array}$ \\
\hline Neurology speciality & 0 & 0 & $1(25)$ & $2(40)$ & $3(5)$ \\
\hline Emergency medicine & $10(36)$ & $6(32)$ & 0 & $3(60)$ & $19(34)$ \\
\hline Non-neurology speciality & $20(71)$ & $18(95)$ & $3(75)$ & $2(40)$ & $43(77)$ \\
\hline
\end{tabular}

Departments responsible for initial management of paediatric head trauma patients for each hospital size and

24 total. Responsible speciality is categorized as neurology speciality (paediatrics; paediatric neurology; neurology; internal medicine), non-neurology (paediatric and general surgery; orthopaedics) or emergency 
medicine. For respective type of speciality, respondent was asked to rate on a 5-grade scale (always; often; sometimes; rarely; never) how frequent this speciality manages children with head trauma at their emergency department. To further simplify presentation, grade "always" and "often" was merged (implying the "most common" clinic/speciality manging children with TBI) and presented for respective hospital size. This means that if grade "sometimes", "rarely" or "never" was chosen for a speciality it won't be presented in the table. Merging of specialities and response options means that the aggregated total response rate won't be $100 \%$. Percentages are calculated as number of responses per total hospitals in each category. Example: There was in total 20 responses in the non-neurology category deriving from local hospitals, implying that in 20 of the 28 local hospitals (71\%) it is common ("often" or "always") that non-neurology physicians are managing children with TBI.

15 hospitals (27\%) reported that these patients are occasionally discharged by a nurse at triage without any doctor assessment. This management was more common $(p<0.001)$ in university and children's hospitals $(n=6 / 9)$ when compared to local and regional hospitals (n $=9 / 47) .8$ of these units had written guidelines concerning this procedure; 6 of these used the SNC16 guidelines.

Almost all hospitals $(n=54)$ use CT as the primary choice of radiology modality to exclude intracranial complications. $96 \%$ of hospitals (54/56) reported full accessibility to a CT scan

20 irrespective of time or day. $48 \%$ of hospitals (27/56) reported routine use of dose-reduction 21 programs. However, many respondents $(23 / 56,41 \%)$ were not aware of any dose-reduction protocol, with only a few hospitals $(2 / 56,4 \%)$ reporting that a dose-reduction protocol was not routinely used and 3 respondents $(5 \%)$ did not answer the question at all. If needed during

24 the head CT scan, $50 \%$ of the respondents reported occasional use of sedation in some form.

25 All hospitals had access to anaesthesiologists irrespective of time or day. 
1 In $75 \%$ of the hospitals (42/56), it was possible to admit patients for in-hospital observation.

2 Most commonly (64\%), these children were admitted to a general ward. In $46 \%(13 / 28)$ of

3 smaller, local hospitals, children could not be admitted in-house and needed to be transferred

4 to another hospital if admission was necessary, see Table 6 for details. In local hospitals, 50\%

$5(7 / 14)$ of children were observed in a non-paediatric ward. In larger hospitals, most children

6 were admitted to paediatric wards $(96 \%, 25 / 26)$. In one hospital, the Intensive Care Unit

7 (ICU) was used for observation.

8

9 Table 6. Possibility of in-hospital observation

\begin{tabular}{|lll|lll|} 
& $\begin{array}{l}\text { Local } \\
\text { Hospital } \\
n(\%)\end{array}$ & $\begin{array}{l}\text { Regional } \\
\text { Hospital } \\
n(\%)\end{array}$ & $\begin{array}{l}\text { Children's } \\
\text { Hospital } \\
n(\%)\end{array}$ & $\begin{array}{l}\text { University } \\
\text { Hospital } \\
n(\%)\end{array}$ & Total \\
\hline $\begin{array}{l}\text { Possibility of } \\
\text { in-hospital observation }\end{array}$ & $15(54)$ & $18(95)$ & $4(100)$ & $5(100)$ & $42(75)$ \\
\hline $\begin{array}{l}\text { No possibility } \\
\text { of in-hospital observation }\end{array}$ & $13(46)$ & $1(5)$ & 0 & 0 & $14(25)$ \\
\hline
\end{tabular}

Numbers presented for respective hospital category and all hospital categories in total.

13 During the observation period, level of consciousness was the most frequently evaluated

14 parameter (95\%), followed by pupillary reaction, heart rate and neurological deficits (see

15 figure 2).

16

17 Different scales were used for evaluating the level of consciousness; the Reaction Level Scale

18 (RLS 85) [26], Glasgow Coma Scale (GCS) or its paediatric version, and the Alert-Verbal-

19 Pain-Unresponsive-scale (AVPU). RLS 85 was the most frequently reported answer, either

20 alone (48\%) or in combination with other assessment scales (29\%) (Figure 3). 
1 Figure 2. Parameters evaluated during in-hospital observation due to paediatric head

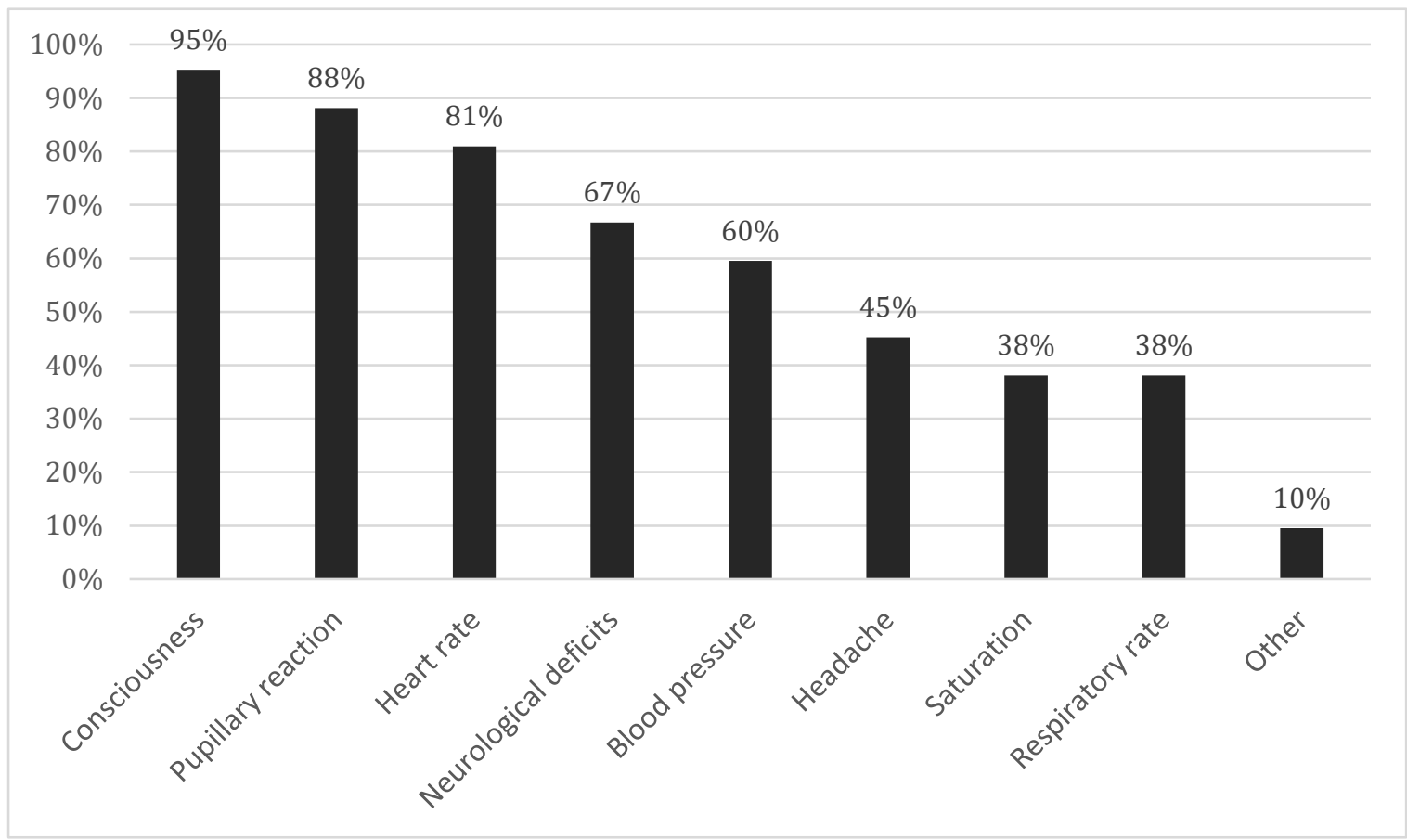

3

$4 \quad$ Respondents could choose more than one alternative $\left(n_{t o t}=42\right)$

5

$6 \quad$ Figure 3. Scale used for assessment of level of consciousness

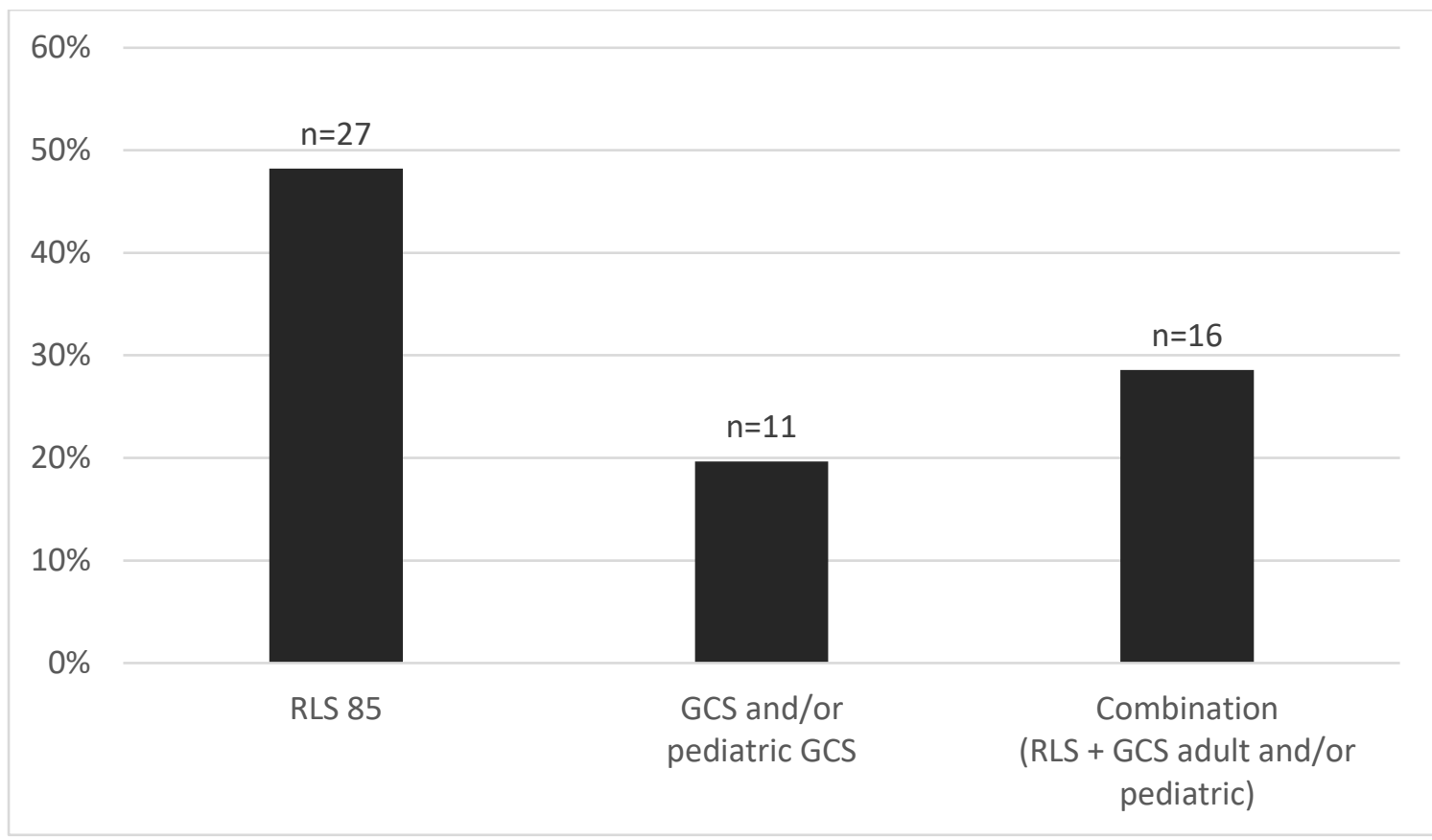

8 Respondents were asked to report which scale(s) that was used at their hospital for assessment of level of 
consciousness. Mmore than one alternative could be marked. RLS-85, GCS (adult version) and GCS (paediatric version) was prespecified options. Three ( $n=3)$ respondents reported use of AVPU-scale in addition to GCS or $R L S-85$.

$597 \%$ of hospitals with capacity for in-hospital admittance (41/42) reported their observation

6 routines. In $46 \%(19 / 41)$ elements of the SNC 16 routines were used regarding type,

7 frequency and/or duration of observation and in 32\% (13/41) the SNC16 was the sole guiding

8 routine for in-hospital management. 44\% (18/41) of the hospitals allowed individual doctor

9 prescription of observation criteria, and in 27\% (11/41) no other routines other than doctor

10 prescribed observation was used. None of the responding hospitals reported a complete lack

11 of routines for observation.

13 Routines for CT scanning in admitted children were mostly (solely or in combination with 14 other written routine) based upon doctor discretion (65\%, 27/41). In 49\% (20/41) of the 15 hospitals a written routine in some form guided CT scanning in admitted children, mainly the $16 \operatorname{SNC16}(31 \%, 13 / 41)$.

$56 \%(23 / 41)$ of hospitals reported lack of discharge-criteria following observation for TBI. Concerning information to patients/guardians at discharge, $15 \%(8 / 55)$ provided only written 20 information, $9 \%(5 / 55)$ provided only oral information and $71 \%(39 / 55)$ provided both, with 21 only 5\% (3/55) not providing discharge information at all.

$2238 \%(21 / 56)$ of all hospitals could arrange a follow up assessment if needed, which was 23 relatively more common at large hospitals $(7 / 9,78 \%$ vs $14 / 47,30 \%)$ and usually either at a 24 paediatric outpatient clinic (52\%) and/or in the primary care sector (48\%). 46\% (26/56) did not provide or plan follow-up in children following TBI; this was more common in small 
1 hospitals (25/26). There was a significant difference in follow-up routines between small

2 (local and regional) and larger (university and children's) hospitals $(\mathrm{p}=0.015)$.

4 In the 2006 survey, 51 hospitals were identified that managed children with TBI. Of these,

5 only $27 \%(14 / 51)$ used management guidelines. In the current study, significantly more

$6(76 \%, 42 / 55)$ of the hospitals used guidelines $(\mathrm{p}<0.001)$. In the present study, only one

7 hospital (2\%) used an ICU for observation, compared to 10 hospitals (20\%) in 2006

$8(\mathrm{p}=0.020)$. The presence of written observation routines (defined as presence of a local

9 written routine regarding in-hospital observation and/or use of the SNC16 recommendation

10 for in-hospital observation) is more common today when compared to 2006 (69\% compared

11 to $31 \%, \mathrm{p}<0.001)$. Finally, the possibility of follow-up after discharge did not differ between

122006 and the current study $(\mathrm{p}=0.22)$. See table 7 below for details.

13

14 Table 7. TBI management in Swedish hospitals, comparison between 2006 [14] and the

15 present study

\begin{tabular}{|llll|}
\hline $\begin{array}{l}\text { 2006 survey } \\
\text { Åstrand et al } \\
\mathrm{n}(\%)\end{array}$ & Current survey & p-value \\
\hline Using established guidelines & $14 / 51(27 \%)$ & $42 / 55(76 \%)$ & $p<0.001$ \\
\hline ICU as observation unit & $10 / 51(20 \%)$ & $1 / 42(2 \%)$ & $p=0.02$ \\
\hline Written observation routines & $16 / 51(31 \%)$ & $29 / 42(69 \%)$ & $p<0.001$ \\
\hline Possibility of follow-up after discharge & $13 / 51(25 \%)$ & $21 / 56(38 \%)$ & $p=0.22$ \\
\hline
\end{tabular}

16 


\section{Discussion}

2 This national cross-sectional survey aims to describe and analyse the current management of

3 children with TBI in Sweden. With a high response rate (>90\%) we have been able to

4 efficiently collect data concerning different aspects of mTBI management. Most hospitals

$5 \quad(76 \%)$ use an established guideline to aid in management and we did not observe a difference

6 of guideline use between sizes of hospital. Most use the SNC16 guideline and the majority of

7 the remaining hospitals use a locally constructed guideline, either a modification of an

8 established guideline, such as PECARN, or one based on expert opinion.

9

10 In $27 \%$ of the hospitals, in particular larger hospitals, nurses can discharge children with

11 mTBI without any assessment by a doctor. These patients reasonably represent the mildest of

12 injuries and are in 53\% (8/15) of the hospitals discharged using a guideline, mainly the

13 SNC16. If children can reliably be assessed by a nurse and judged to be in the mildest risk

14 group of TBI, this type of management may be efficient. Further studies could evaluate this

15 issue.

16

17 Children with TBI were managed predominately by non-specialists and (outside of dedicated

18 children's hospitals) non-paediatricians. These findings reinforce the need of a nationally

19 implemented and accepted guideline for these patients, as most children with TBI will be

20 managed by inexperienced doctors from varying specialities, especially in smaller hospitals.

21 The field of emergency medicine is relatively young in Sweden, but may be the primary

22 group to manage TBI patients in the future. 
1 Sweden is not a densely populated country with some concentrations of inhabitants in larger

2 cities. Due to this fact, large university hospitals are generally located in areas where many

3 inhabitants reside. Large parts of Sweden are therefore some distance from these hospitals

4 which contain the neurosurgery departments. Indeed, $16 \%$ of hospitals reported having at

5 least 2 hours transfer time to the nearest neurosurgical unit. This aspect is important, as the

6 severe complications after mTBI, although uncommon [9,27], require immediate attention

7 and often neurosurgical expertise.

8

9 In approximately half $(13 / 28,46 \%)$ of local hospitals, there was no possibility of in-hospital

10 observation of children with mTBI. These children are instead sent to adjacent, larger

11 hospitals, indicating a practical and logistical hurdle for guideline development. The most common parameter evaluated during observation is level of consciousness, followed by simple measures of neurological function, such as pupillary reaction. Concerning observation routines, most hospitals had written routines for which parameters should be measured, including how often and for how long, and approximately half of these used the SNC16 observation routines.

Due to the potential risk of ionizing radiation in children, the aspect of dose-reduction protocols is highly relevant. Although approximately half of hospitals stated that routine reduction of radiation dose was used, many respondents could not reliably answer this question. In addition to the radiology departments, the referring party must also be made aware of the radiation issues related to CT scans [17]. In order to fully investigate this issue, a survey directed at the radiological department of the hospitals is warranted.

24 Following TBI, children may be manged (with CT, in-hospital observation, or both) in order to detect possible severe complications. In the absence of these, these children may be 
1 discharged. The criteria for discharge may vary between hospitals but usually include the

2 absence of worrying signs and symptoms. More than half of hospitals did not have specific

3 discharge criteria for these patients. Although evidence is lacking in this area, written

4 discharge criteria may facilitate management and promote equality in patient management.

5 Discharge from the hospital, be that after initial assessment in the ED or after in-hospital

6 observation, should be accompanied with information regarding the injury, what to expect

7 and when to seek health care. Pleasingly, most hospitals provided this with only 3 hospitals

$8 \quad(5 \%)$ stating that they do not provide such information.

10 A similar survey was conducted 2004 - 2005 and published 2006 [14] by our group and one 11 major aim of this study was to analyse any change of management over time. During this 12 time period, several high-quality CDR's and guidelines have been published $[9,19,20]$. A

13 guideline of special interest in the Swedish context is the Scandinavian guideline [5]

14 published in 2016, with a published summary in Swedish in Läkartidningen [28] in 2017.

15 This CDR was developed to give more precise recommendations on observation as an option

16 to head CT, with a stepwise increase in the duration of observation based on a risk

17 stratification in five groups. It also suggests an absolute or relative need for a head CT

18 secondary to the risk stratification, recommendations for in-hospital observation routines and

19 discharge information.

21 A significant increase in guideline use has occurred since 2006 (76\% vs $27 \%$ ), where more than half of these hospitals use the SNC16 guideline. The use of formal, written observation routines are more common today than in 2006, although almost a third of the hospitals still

24 haven't formalised this part of paediatric TBI management. The widespread use of elements 25 in the SNC16 guideline, including other aspects than the risk criteria, such as observation 
1 routines, in Sweden is gratifying as it implies introduction of a sound evidence-based

2 approach in the management of head injuries in children. As these guidelines are still to be

3 internally validated in the Scandinavian setting a certain amount of vigilance is still required,

4 although external validation has shown encouraging results [22].

6 The strengths of this study lie in the high response rate and the on-line survey system which

7 increases response accuracy and minimises ambiguous answers. As this group did a similar

8 survey in 2006, most questions are similar which allows a comparison over time. This study

9 has several limitations. Despite our best efforts to ensure that the respondent was fully aware

10 of all aspects of TBI management at their respective hospital, inaccuracies may still have

11 occurred. Also, the questions response rate was not always $100 \%$ within the same hospital

12 which may also account for some errors. However, these issues were minimal and have little

13 effect of the overall results.

15 In a survey such as this, there is always a balance between the complexity of the survey

16 material and response compliance. Some areas, such as the questions concerning dose-

17 reduction of CT scans, warrant further investigation in a new, separate survey to a new set of

18 respondents more likely to have local knowledge concerning this aspect. Concerning the

19 results of this study, there seems to be a widespread adoption and use of the SNC16

20 guideline. However, many hospitals still use management methods based upon weaker

21 scientific methods, such as management based upon local expert opinion. Strategies and

22 further research aiming to facilitate implementation of new evidence in emergency

23 departments is important and needed. However, today's management was generally better

24 when compared to management derived from the 2006 survey. Finally, validation of a 
1 guideline should be encouraged before widespread clinical use. Efforts to increase

2 participation in an ongoing Nordic validation study are warranted.

\section{Conclusions}

4 Management of children with TBI varies in Sweden, although many aspects have improved

5 over the last 15 years. Most hospitals use established guidelines, utilise dose-reduction

6 protocols for $\mathrm{CT}$, use written observation routines and provide adequate information to

7 patients/guardians at discharge. A minority of hospitals use management routines not based

8 upon scientific evidence.

\section{Declarations}

10 Ethics approval and consent to participate

11 The study does not include individual patient data. Ethics approval by the Swedish Ethical

12 Review Authority, Dnr 2020-02693

13 Consent for publication

14 Not applicable.

15 Availability of data and materials

16 The datasets used and/or analysed during the current study are available from the

17 corresponding author on reasonable request. 


\section{$1 \quad$ Competing interests}

2 None of the authors have any financial competing interests. JU is a member of the SNC

3 committee, a non-profit organisation independent from financial company support, who are

4 responsible for the SNC16 guidelines.

\section{$5 \quad$ Funding}

6 This study was funded by Södra Sjukvårdsregionen and Vetenskapliga Rådet, Hallands

7 Sjukhus.

\section{Authors' contributions}

9 JU och FW conceived and planned the study. LP made the survey and contacted respondents.

10 LP summarised the results and wrote the first draft together with FW and JU. All authors

11 have read the manuscript.

\section{Acknowledgements}

13 We would like to thank Region Halland for ongoing support with research efforts, especially

14 the FoU department, including Anders Holmén and Amir Bagir. 


\section{References}

2 [1]ARAKI T, YOKOTA H, MORITA A. Pediatric Traumatic Brain Injury: Characteristic

3 Features, Diagnosis, and Management. Neurologia medico-chirurgica 2017;57(2):82-93.

4 [2]Thurman DJ. The Epidemiology of Traumatic Brain Injury in Children and Youths: A

5 Review of Research Since 1990. J Child Neurol 2016 January 01;31(1):20-27.

6 [3]Babl FE, Lyttle MD, Bressan S, Borland M, Phillips N, Kochar A, et al. A prospective

7 observational study to assess the diagnostic accuracy of clinical decision rules for children

8 presenting to emergency departments after head injuries (protocol): the Australasian

9 Paediatric Head Injury Rules Study (APHIRST). BMC pediatrics 2014;14(1):148.

10 [4]Cassidy JD, Carroll LJ, Peloso PM, Borg J, von Holst H, Holm L, et al. Incidence, risk

11 factors and prevention of mild traumatic brain injury: results of the WHO Collaborating

12 Centre Task Force on Mild Traumatic Brain Injury. J Rehabil Med 2004(43 Suppl):28-60.

13 [5]Ramona Astrand, Christina Rosenlund, Johan Unden. Scandinavian guidelines for initial

14 management of minor and moderate head trauma in children. BMC Medicine 2016 Jan

$151, ; 14(1): 33$.

16 [6]Feigin VL, Theadom A, Barker-Collo S, Starkey NJ, McPherson K, Kahan M, et al.

17 Incidence of traumatic brain injury in New Zealand: a population-based study. Lancet Neurol

182013 January 01;12(1):53-64.

19 [7]Babcock L, Byczkowski T, Wade SL, Ho M, Mookerjee S, Bazarian JJ. Predicting

20 postconcussion syndrome after mild traumatic brain injury in children and adolescents who

21 present to the emergency department. JAMA Pediatr 2013 February 01;167(2):156-161. 
1 [8]Barlow M, Schlabach D, Peiffer J, Cook C. Differences in change scores and the

2 predictive validity of three commonly used measures following concussion in the middle

3 school and high school aged population. Int J Sports Phys Ther 2011 September 01;6(3):150-

4157.

5 [9]Kuppermann N, Holmes JF, Dayan PS, Hoyle JD Jr, Atabaki SM, Holubkov R, et al.

6 Identification of children at very low risk of clinically-important brain injuries after head

7 trauma: a prospective cohort study. The Lancet 2009;374(9696):1160-70.

8 [10]Pearce MS, Salotti JA, Little MP, McHugh K, Lee C, Kim KP, et al. Radiation exposure

9 from CT scans in childhood and subsequent risk of leukaemia and brain tumours: a

10 retrospective cohort study. Lancet 2012 August 04;380(9840):499-505.

11 [11]Brenner D, Elliston C, Hall E, Berdon W. Estimated risks of radiation-induced fatal

12 cancer from pediatric CT. AJR Am J Roentgenol 2001 February 01;176(2):289-296.

13 [12]Bernier MO, Rehel JL, Brisse HJ, Wu-Zhou X, Caer-Lorho S, Jacob S, et al. Radiation

14 exposure from CT in early childhood: a French large-scale multicentre study. Br J Radiol

152012 January 01;85(1009):53-60.

16 [13]af Geijerstam JL, Oredsson S, Britton M, OCTOPUS Study Investigators. Medical

17 outcome after immediate computed tomography or admission for observation in patients with

18 mild head injury: randomised controlled trial. BMJ 2006 September 02;333(7566):465.

19 [14]Astrand R, Unden J, Bellner J, Romner B. Survey of the management of children with

20 minor head injuries in Sweden. Acta Neurol Scand 2006 April 01;113(4):262-266. 
1 [15]Cheng C, Pan H, Li C, Chen Y, Chen C, Huang Y, et al. Physicians' Risk Tolerance and

2 Head Computed Tomography Use for Pediatric Patients With Minor Head Injury. Pediatric

3 emergency care 2018 May 25,;37(3):e129-e135.

4 [16]Griffey RT, Jeffe DB, Bailey T. Emergency physicians' attitudes and preferences

5 regarding computed tomography, radiation exposure, and imaging decision support. Acad

$6 \quad$ Emerg Med 2014 July 01;21(7):768-777.

7 [17]Jorulf Håkan, Isberg Bengt, Svahn Ulla. Radiologiska undersökningar av barn - en studie

8 av metodval. En nationell kartläggning av berättigande, metodval och remisskvalitet.

9 Strålsäkerhetsmyndigheten $2015: 26$.

10 [18]Elmoheen A, Salem W, Bashir K. Reducing unnecessary CT scan of the head for minor

11 paediatric head injuries at the emergency department. BMJ Open Qual 2021 January

$1201 ; 10(1): 10.1136 /$ bmjoq-000973.

13 [19]Osmond MH, Klassen TP, Wells GA, Correll R, Jarvis A, Joubert G, et al. CATCH: a

14 clinical decision rule for the use of computed tomography in children with minor head injury.

15 Canadian Medical Association Journal 2010 Mar 9,;182(4):341-348.

16 [20]Dunning J, Daly JP, Lomas J, Lecky F, Batchelor J, Mackway-Jones K. Derivation of the

17 children's head injury algorithm for the prediction of important clinical events decision rule

18 for head injury in children. Archives of disease in childhood 2006 Nov;91(11):885-891.

19 [21]Ingebrigtsen T, Romner B, Kock-Jensen C. Scandinavian guidelines for initial

20 management of minimal, mild, and moderate head injuries. The Scandinavian Neurotrauma

21 Committee. Journal of Trauma-Injury Infection \& Critical Care 2000;48(4):760-6. 
1 [22]Undén J, Dalziel SR, Borland ML, Phillips N, Kochar A, Lyttle MD, et al. External

2 validation of the Scandinavian guidelines for management of minimal, mild and moderate

3 head injuries in children. BMC Medicine 2018;16(1).

4 [23]Babl FE, Tavender E, Ballard DW, Borland ML, Oakley E, Cotterell E, et al. Australian 5 and New Zealand Guideline for Mild to Moderate Head Injuries in Children. Emerg Med 6 Australas 2021 April 01;33(2):214-231.

7 [24]Vedin T, Edelhamre M, Karlsson M, Bergenheim M, Larsson PA. Management of 8 Traumatic Brain Injury in the Emergency Department: Guideline Adherence and Patient 9 Safety. Qual Manag Health Care 2017 December 01;26(4):190-195.

10 [25]Donnell Z, Hoffman R, Myers G, Sarmiento K. Seeking to improve care for young 11 patients: Development of tools to support the implementation of the CDC Pediatric mTBI 12 Guideline. J Safety Res 2018 December 01;67:203-209.

13 [26]Starmark JE, Stålhammar D, Holmgren E. The Reaction Level Scale (RLS85). Manual and guidelines. Acta Neurochir (Wien) 1988;91(1-2):12-20.

[27]Klassen TP, Reed MH, Stiell IG, Nijssen-Jordan C, Tenenbein M, Joubert G, et al.

16 Variation in utilization of computed tomography scanning for the investigation of minor head

17 trauma in children: a Canadian experience. Academic emergency medicine : official journal

18 of the Society for Academic Emergency Medicine 2000;7(7):739-44.

19 [28]Olivecrona Z, Winberg H, Lannge M, Undén J. Nya skandinaviska riktlinjer för att 20 handlägga skallskador hos barn. Evidens- och konsensusbaserade rekommendationer för 21 minimala, lätta och medelsvåra skador. Läkartidningen $2017,114: E F M Z ; 15-16 / 2017$. 